\title{
Toxic additives in medication for preterm infants
}

\author{
A Whittaker, ${ }^{1}$ A E Currie, ${ }^{1}$ M A Turner, ${ }^{2}$ D J Field,${ }^{3} \mathrm{H}$ Mulla, ${ }^{4} \mathrm{H}$ C Pandya ${ }^{5}$
}

${ }^{1}$ Neonatal Unit, Leicester Royal Infirmary, University Hospitals Leicester NHS Trust, Leicester, UK; ${ }^{2}$ Division of Perinatal and Developmental Medicine, University of Liverpool, Liverpool, UK; ${ }^{3}$ Department of Health Sciences, University of Leicester, Leicester, UK; ${ }^{4}$ Centre for Therapeutic Evaluation of Drugs in Children, University Hospitals Leicester NHS; ${ }^{5}$ Department of Infection, Immunity \& Inflammation,

University of Leicester, Leicester, UK

Correspondence to:

Dr H C Pandya, Department of Infection, Immunity \& Inflammation, University of Leicester Robert Kilpatrick Clinical Sciences Building, Leicester Royal Infirmary, Leicester LE2 7LX, UK; hp28@ le.ac.uk

Accepted 21 October 2008 Published Online First 21 January 2009

\section{ABSTRACT}

Background: Little is known about exposure of preterm infants to excipients during routine clinical care.

Objective: To document excipient exposure in vulnerable preterm babies in a single centre, taking into account chronic lung disease (CLD) as a marker of illness severity. Design: Excipient exposure after treatment with eight oral liquid medications was determined by retrospectively analysing the drug charts of infants admitted to a

neonatal unit.

Setting: The Leicester Neonatal Service.

Participants: 38 infants born between June 2005 and July 2006 who were less than 30 weeks' gestation and $1500 \mathrm{~g}$ in weight at birth and managed in Leicester to discharge.

Results: The 38 infants represented $53 \%$ of the eligible target group; 7/38 infants had CLD. During their in-patient stay, infants were exposed to over 20 excipients including ethanol and propylene glycol, chemicals associated with neurotoxicity. Infants with CLD were exposed to higher concentrations of these toxins. Infants were also exposed to high concentrations of sorbitol, with some infants being exposed to concentrations in excess of recommended guidelines for maximum exposure in adults.

Conclusions: Preterm infants are commonly exposed to excipients, some of which are potentially toxic. Strategies aimed at reducing excipient load in preterm infants are urgently required

Children born prematurely are at risk of developing a variety of problems ranging from anaemia of prematurity and neonatal bone disease to patent ductus arteriosus (PDA) and chronic lung disease of prematurity (CLD). ${ }^{1}$ As babies cannot take tablets, it is common practice to prevent and/or treat these complications with intravenous and oral liquid medications such as iron, vitamin and mineral supplements, and drugs such as furosemide and dexamethasone. ${ }^{1}$ Although there are some data on the safety, efficacy, pharmacokinetics and potential interactions of these agents in premature babies, little is known about the safety of the excipients (solvents, vehicles, emulsifiers and preservatives) used in formulating oral liquid preparations of these drugs.

Most intravenous (IV) drug formulations are relatively simple, containing drug, solvent (commonly water) and $\mathrm{pH}$-adjusting agents. Toxicity concerns often preclude non-water solvents being used to formulate water-insoluble drugs for IV administration. The other main features of IV formulations are linked to the manufacturing processes. IV drug preparations can often be "terminally sterilised" by autoclaving. Packaging the preparation in air-tight and sometimes air-free containers maintains drug sterility and enhances shelf-life. ${ }^{2}$ However, once opened, the sterility and

\section{What is already known on this topic}

- Excipients in oral liquid medications may be toxic to humans.

- Cumulative exposure is one of several factors that determine whether a given excipient is likely to have toxic effects.

\section{What this study adds}

- This study provides a preliminary estimate of the problem of excipient exposure in preterm infants.

- The findings suggest that significant exposure to potentially harmful excipients (eg, ethanol) is common.

stability of the preparation cannot be maintained. Thus, most IV drugs are marked "for single use only", ie, any excess drug must be discarded shortly after the container has been opened.

The issues relating to oral liquid medications are quite different. The gastrointestinal (GI) tract is a physical barrier which can limit systemic absorption of chemical agents. This factor, along with the "detoxifying" actions of the upper GI tract and liver, allows manufacturers to add chemicals to oral liquid medicines that would be toxic if administered by the IV route. Thus, if necessary, manufacturers will use solvents other than water, eg ethanol, to produce oral liquid medicines. To increase palatability and thereby compliance, manufacturers will also often add colourings, sweeteners and flavourings to the preparation. ${ }^{2}{ }^{3}$ In addition, as oral liquid medications are generally not "terminally sterilised", they may contain preservatives. These chemicals increase the shelflife of the drug. Hence, oral liquid preparations are the most common route by which patients are exposed to excipients in drugs.

In oral liquid formulations used for children, manufacturers generally rely on data gathered from animal and adult human studies. There are few direct data on the safety of specific excipients in preterm infants. ${ }^{3}$ The European Medicines Agency (EMEA) has recently drawn attention to the need to consider excipients in the assessment of medicines' safety in newborns. ${ }^{4}$ To our knowledge, there are no data about the extent to which premature neonates are exposed to excipients. In this study, we sought to derive preliminary estimates of excipient exposure in a group of preterm infants during their stay in a tertiary 
Table 1 Basic details of infants included in the study

\begin{tabular}{lcc}
\hline & $\begin{array}{l}\text { No dexamethasone } \\
(\mathbf{n}=\mathbf{3 1})\end{array}$ & $\begin{array}{l}\text { Dexamethasone } \\
(\mathbf{n}=\mathbf{7})\end{array}$ \\
\hline Male/female & $17 / 13$ & $5 / 2$ \\
GA at birth (weeks) & $27^{+4}$ & $26^{+3}$ \\
Weight (g)* & $1052(298)$ & $813(205)$ \\
CGA at discharge (weeks) & $38^{+3}$ & $42^{+4}$ \\
Duration of ventilation (days)* & $10.7(9.9)$ & $24.1(16.5)$ \\
Days in oxygen* & $46.5(34.4)$ & $57.3(53.6)$ \\
Number oxygen-dependent & & \\
$\quad 28$ days PNA & 25 & 7 \\
36 weeks GA & 15 & 7 \\
\hline
\end{tabular}

*Mean (SD).

CGA, corrected gestational age; PNA, postnatal age; GA, gestational age.

referral unit. In addition, because of recent concern about the potential role of excipients in the development of CLD, ${ }^{5}$ we also assessed specifically whether babies with this condition and treated with dexamethasone experienced a different pattern of excipient exposure.

\section{METHODS}

\section{Sample population}

Preterm infants born at or below 30 weeks' gestation and weighing less than $1500 \mathrm{~g}$ who survived to discharge who spent their entire in-patient admission in the neonatal units of University Hospitals Leicester NHS Trust were eligible for inclusion in the study. These inclusion criteria meant that each baby had estimates of weekly excipient exposure made for a minimum of 4 weeks and that problems relating to incomplete data or inter-hospital changes in formulations were avoided.

\section{Drugs included in analysis}

To generate a representative picture of excipient exposure of preterm babies, only commonly used drugs were included in this study. These criteria yielded iron, vitamin drops, domperidone and caffeine. In the Leicester Neonatal Service, the most common drugs used in the early management of CLD are dexamethasone, spironolactone, bendrofluazide and furosemide. To assess excipient exposure in babies with this common condition, these were also included in the study.

\section{Study period and data collection}

The notes of eligible infants admitted between June 2005 and July 2006 inclusive were analysed if they were complete and available during April-June 2007. Details on sex, gestation, weight at birth, weekly weight, the clinical course, daily

Table 2 Mean duration of drug treatment for sample population separated according to whether or not infants received treatment with dexamethasone

\begin{tabular}{llll}
\hline \multirow{2}{*}{ Drug } & \multirow{2}{*}{$\begin{array}{l}\text { No of group } \\
\text { given drug }\end{array}$} & \multicolumn{2}{l}{ Length of therapy (weeks) } \\
\cline { 3 - 4 } & 38 & No dexamethasone & Dexamethasone \\
\hline Iron & 36 & 8.3 & 9.0 \\
Vitamin drops & 36 & 8.7 & 7.7 \\
Caffeine & 17 & 5.7 & 3.8 \\
Domperidone & 12 & 2.6 & 8.5 \\
Furosemide & 16 & 3.8 & 3.25 \\
Spironolactone & 10 & 4.0 & 2.5 \\
Bendrofluazide & 7 & - & 3.5 \\
Dexamethasone & & & 3.3 \\
\hline
\end{tabular}

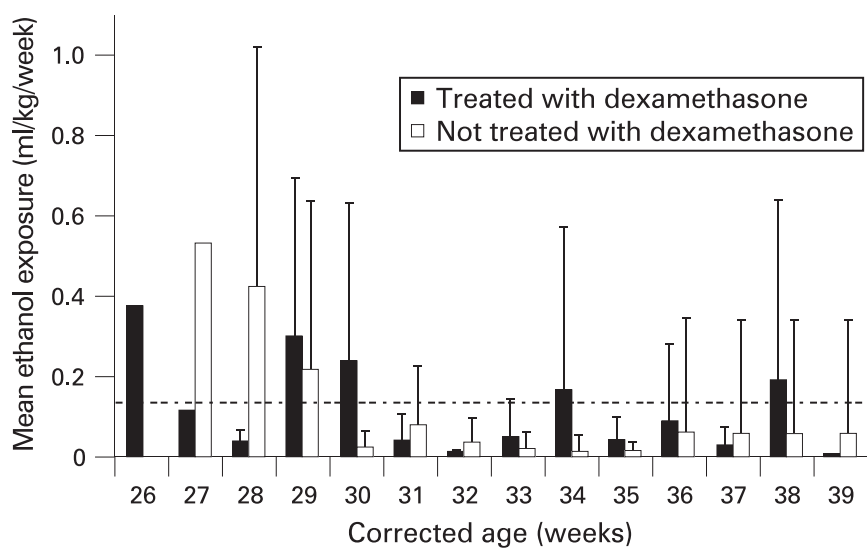

Figure 1 Age-corrected mean (SD) weekly exposure to $96 \%$ ethanol for neonates with chronic lung disease either treated or not treated with dexamethasone. The dotted line ( $=0.14 \mathrm{ml} / \mathrm{kg} /$ week) represents an intake of 1 unit of alcohol per week for an average man weighing $70 \mathrm{~kg}$ (see Discussion).

medication dose, dosing schedule and route of administration of drugs included in the study were recorded on data collection forms. It was also noted whether infants met the Paediatric Consensus recommendations for CLD. ${ }^{6}$ The source of each formulation (and strength) of each drug used during the study period was obtained from pharmacy records. Every manufacturer except the domperidone manufacturer provided data on the exact formulation of their drug.

\section{Data analysis and ethics}

For any given excipient, the amount per $\mathrm{ml}$ in a formulation was calculated using data supplied by the manufacturer. Using drug dose and body weight data, the amount of a specific excipient each baby received was calculated on a "per $\mathrm{kg}$ per week" basis. Summary statistics (mean (SD)) were calculated using Microsoft Excel. Formal ethics advisory committee approval was not required in our institution for this retrospective chart review.

\section{RESULTS}

A total of 71 infants met the inclusion criteria. The medical notes of 45 of these infants were available to the investigators in the time window of the study. However, data from seven infants were excluded because one or more drug charts were missing. The results and discussion presented below relate to the remaining 38 infants. A later examination of a subset of notes (six patients) from the babies excluded from the original group of 71 because their notes were unavailable at the correct time identified no major therapeutic differences from the group that were included in the analysis (data not shown). Table 1 summarises the basic details of babies included in the analysis and groups of infants according to whether they had CLD and received dexamethasone therapy.

A large number of excipients were present in the oral liquid formulations of the eight drugs included in this study, with some present in more than one formulation. The excipients in the following formulations were:

(a) Iron: ethanol, hydroxybenzoate, citric acid, Ponceau 4R (E124), black cherry, glycerol, saccharin and sorbitol

(b) Vitamin drops: hydroxybenzoate, sodium hydroxide, sucrose and polysorbate 20

(c) Caffeine: hydroxybenzoate 
(d) Domperidone: hydroxybenzoate, sodium hydroxide, saccharin, cellulose and polysorbate 20

(e) Furosemide: ethanol, cherry flavour and malitol

(f) Spironolactone: hydroxybenzoate, raspberry flavour, aspartame and xanthum gum

(g) Bendrofluazide: hydroxybenzoate and xanthan gum

(h) Dexamethasone: benzoic acid, propylene glycol (PG), mint flavour, malitol and sorbitol

Table 2 shows the mean duration of drug therapy. Ethanol (96\%) was found in several medications. Exposure to ethanol ranged from $0.2 \mathrm{ml}$ to $1.8 \mathrm{ml}$ at each week of corrected age (fig 1). Ethanol exposure between 27 and 29 weeks' corrected age was similar in all infants irrespective of whether they received dexamethasone therapy for CLD. However, the data suggest that exposure to ethanol remained static over the next 10 weeks for those not treated with dexamethasone, whereas infants receiving dexamethasone treatment had a greater weekly exposure to this excipient during this period.

Sorbitol was also a common excipient. Figure 2 shows the sorbitol exposure for each week of corrected age. Exposure to sorbitol ranged from 0.1 to $3.5 \mathrm{~g} / \mathrm{kg} /$ week. The recommended maximal intake of sorbitol in adults is $20 \mathrm{~g} /$ day, although studies show that sorbitol-induced GI side effects may occur at much lower intakes.7 Using the weight of an "average" adult male $(70 \mathrm{~kg})$ as the denominator, the adult recommendations equate to $2 \mathrm{~g}$ of sorbitol $/ \mathrm{kg}$ body weight/week (dotted line, fig 2). Eighteen infants exceeded this threshold during at least 1 week. Only infants given dexamethasone were exposed to PG. The World Health Organization has set an acceptable daily intake for this excipient at up to $25 \mathrm{mg} / \mathrm{kg}$ body weight $(175 \mathrm{mg} / \mathrm{kg} / \text { week })^{8}$ Figure 3 shows that all infants given dexamethasone exceeded this limit at some stage.

\section{DISCUSSION}

The effects of any excipient on health can only be determined by characterising its exposure in various patient groups. The list of excipients to which preterm neonates may be exposed during their stay in intensive care units is likely to be larger than presented herein, as not all drugs used by the Leicester service, eg, nystatin and omeprazole, were included in this study.

Although it is recognised that oral liquid medications containing excipients are given to preterm infants, there are few direct data on their safety. The results presented here will

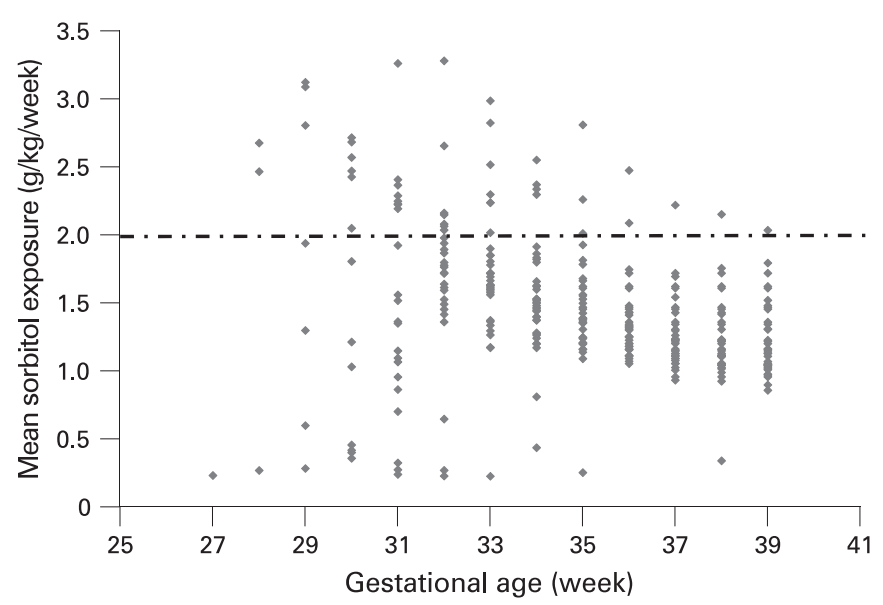

Figure 2 Age-corrected mean weekly exposure to sorbitol for each "neonate-week". The dotted line refers to a "recommended limit" $(2$ g/kg week) derived from extrapolating safe limits for adults.

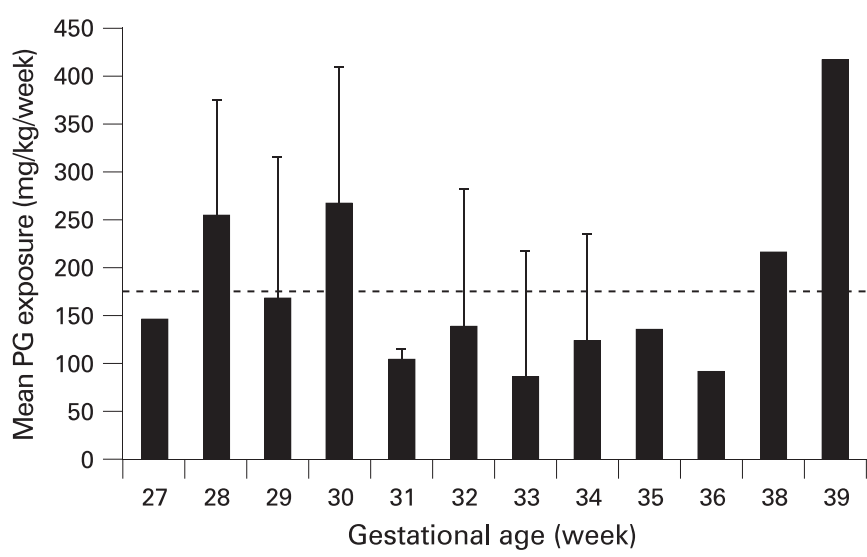

Figure 3 Age-corrected mean (SD) weekly exposure to propylene glycol (PG) in infants receiving dexamethasone treatment. The dotted line represents WHO recommended limit for adults (175 mg/kg/week).

understandably generate concern among parents, nurses, pharmacists and paediatricians. It is important to stress that no clinical link between excipient exposure and outcomes has been made as a result of this investigation.

Iron, multivitamins and folic acid are a group of oral liquid medications commonly prescribed to preterm infants for 36 months. Therefore the excipients contained in these medications form a "background" level of excipient exposure, which many preterm infants experience during early life. Oral liquid medications such as furosemide and dexamethasone are given to some infants to manage relatively common and potentially lifethreatening complications of preterm birth, such as CLD and PDA. Currently, withholding the drug is the only means of avoiding exposure to the excipients contained within these medications.

Excipients in oral liquid medications are added for good clinical and or manufacturing reasons. Some are essential for drug solubility (eg, ethanol, PG), whereas others act to prevent microbial contamination (eg, parabens, benzoic acid) or in some cases buffer the $\mathrm{pH}$ of the liquid (sodium hydroxide). However, it is not immediately apparent why formulations given to infants primarily fed through nasogastric tubes contain colourings and flavourings. Many agents in this group of excipients are considered harmless as they are derived from natural sources. One potential exception to this rule is Ponceau 4R, a colouring agent banned in some countries because of concerns about its effects on neurocognitive development and behaviour. Indeed, following work by Eigenmann and Haenggeli, ${ }^{9}$ the United Kingdom Food Standards Agency (FSA) has recently recommended that Ponceau $4 \mathrm{R}$ and some other colouring agents be removed from foods altogether because of these concerns. ${ }^{10}$ Although it is reassuring that no infant (data not shown) was exposed to more than the recommended intake levels for adults (>4 mg/kg/day), ${ }^{11}{ }^{12}$ we propose that Ponceau 4R should also be removed from all medications because of these concerns.

Although it is difficult to justify the use of any colouring, sweetener and flavouring in products given to infants who are not yet feeding orally, it is also important to recognise that oral formulations have a wide use that includes school-aged children and older people, as well as preterm infants. In these other patient groups, many excipients are used to improve tolerability and hence medication adherence. Manufacturers may also argue that commercial pressures mean that they are only able to produce a "one fits all" formulation of a drug. However, many 
thousands of doses of these medications are administered each year, and it is also possible that commercial enterprises have not explored this market adequately.

As preterm infants in our unit were exposed to over 20 excipients, exposure data for all the excipients are not presented for purposes of clarity. Our findings suggest that preterm infants are exposed to ethanol in two ways. The first is chronic low-level exposure following administration of iron, and the second is high-level exposure secondary to furosemide treatment. Several infants received relatively short courses of oral furosemide treatment 1-3 weeks after birth to manage PDA. However, a number of children received long courses of oral furosemide therapy to manage an unresolved PDA (one case) or as an adjunct to dexamethasone treatment in managing CLD.

It is accepted that infants should not be exposed to ethanol. For adults of average body weight and with normal liver function, the "recommended safe weekly limits" of ethanol consumption are 14 units for women and 21 units for men (1 unit of alcohol $=10 \mathrm{ml} 96 \%$ ethanol). ${ }^{13}$ Thus, assuming that an average man weighs $70 \mathrm{~kg}$, ethanol exposure should not exceed $3 \mathrm{ml} / \mathrm{kg}$ body weight/week. Ethanol exposure in the preterm infants included in this study ranged from 0.2 to $1.8 \mathrm{ml} /$ week, uncorrected for weight. As all the infants weighed between 1 and $3.5 \mathrm{~kg}$ (data not shown) during the period of study, our findings suggest that these infants are exposed to significant concentrations of ethanol. In terms of "units of alcohol", we estimate the exposure to lie between 1 and 7 units during some weeks ( 1 unit alcohol/week equates to $0.14 \mathrm{ml}$ ethanol $/ \mathrm{kg}$ body weight/week for a man weighing $70 \mathrm{~kg}$ ).

There is clear evidence that ethanol can adversely alter somatic growth and neuronal migration during fetal development. ${ }^{14}{ }^{15}$ As the safe exposure limit for ethanol is unknown, most authorities advocate a "no alcohol during pregnancy" policy. ${ }^{15}{ }^{16}$ Our findings have a number of clinical implications. Firstly, it is possible that adverse neurological outcome of infants with or without CLD may be due to ethanol exposure. Secondly, the toxicity of active drugs, eg, dexamethasone, may be exacerbated by inadvertent co-administration of ethanol, particularly where the toxic effects occur in the same tissue, eg, the central nervous system. Finally, in the absence of an alternative to an ethanol-containing furosemide formulation, cardiologists and neonatologists need to consider the risk of chronic ethanol administration in deciding how best to manage infants and children with heart failure and CLD.

PG is an alcohol solvent widely used to prepare drug formulations. Like ethanol, it is rapidly absorbed form the GI tract and is metabolised in the liver (to pyruvic acid and lactic acid) and also excreted unchanged in urine. PG is estimated to be one-third as intoxicating as ethanol. ${ }^{8}$ At large doses, adverse central nervous system effects have been reported in infants and children, including seizures and ototoxicity. ${ }^{17-19}$ It is of great concern that many of the infants in this study exceeded the WHO acceptable daily intake limit for PG in adults corrected for weight. ${ }^{20}$ Furthermore, it is important to stress that, because PG is excreted by the kidneys and that renal function is closely related to gestational age, the limit of "up to $25 \mathrm{mg} / \mathrm{kg}$ " is likely to be significantly lower for preterm infants. In addition, as PG is the solvent used to formulate dexamethasone, as with ethanol, their co-administration may significantly lower the toxicity threshold of both.

Sorbitol (D-glucitol) is a hexhydric alcohol (polyol) used widely as an excipient by drug manufacturers. Although there is no maximum safe dose, it is suggested that the maximum daily intake of sorbitol should not exceed $20 \mathrm{~g} /$ day for an average adult. ${ }^{7}$ Many neonates in our cohort exceeded this dose when it was corrected for weight. The reported side effects of sorbitol in young infants (not preterm) include diarrhoea, colic and nutrient malabsorption. ${ }^{21} 22$ These occur because sorbitol absorption in the GI tract is relatively poor. However, sorbitol uptake may be increased when co-ingested with glucose and lipids. ${ }^{23}$ Although fetuses appear to use sorbitol as an energy source in utero, ${ }^{24}{ }^{25}$ little is known about sorbitol metabolism in preterm infants. The known GI effects in infants and the potential role that sorbitol accumulation plays in causing diabetic complications such as retinopathy and cataract ${ }^{26}$ indicates that studies of sorbitol metabolism in preterm infants are needed to define safe exposure limits for this excipient in this group.

This study was designed to yield a preliminary estimate of the problem of excipient exposure in preterm infants managed through an exploratory analysis of routinely collected data. As such it had several limitations. Firstly, we used a sample of convenience; secondly, we examined only a small selection of medications; thirdly, we were unable to obtain detailed information about the constitution of all the medications we studied. However, it is likely that the finding of this study reflect the extent of excipient exposure among neonates in the rest of England or UK, as an unpublished survey of English neonatal units found that many use the same formulations as those used in Leicester. We feel it is important that the EMEA/ Medicines and Healthcare Products Regulatory Agency not only ensures that all manufacturers provide detailed labelling of the excipient content of their products, but also leads action to determine whether existing practice constitutes a risk and, if so, how this might be dealt with.

Competing interests: None.

\section{REFERENCES}

1. Rennie JM (ed). Roberton's textbook of neonatology. 4th edn. Amsterdam: Elsevier, 2005.

2. Swarbrick J, Boylan JC. Excipients. In: Encyclopedia of pharmaceutical technology. 2nd edn. New York: Marcel Dekker Inc, 2002;3:1132-64.

3. Nunn T, Williams J. Formulation of medicines for children. Br J Clin Pharmacol 2005;59:674-6

4. Eutopean Medicines Agency. Neonates workshop: Workshop on regulatory and scientific issues related to the investigation of medicinal products intended for neonatal use. www.emea.europa.eu/pdfs/human/paediatrics/workshops/neonates/ final.pdf (accessed 25 Nov 2008).

5. Walsh MC, Szefler S, Davis J, et al. Summary proceedings from the bronchopulmonary dysplasia group. Pediatrics 2006;117:S52-6.

6. Jobe AH, Bancalari E. Bronchopulmonary dysplasia. Am J Respir Crit Care Med 2001;163:1723-9.

7. Rowe RC, Sheskey PJ, Owen SC (eds). Sorbitol. In: Handbook of pharmaceutical excipients. 5th edn. London: Pharmaceutical Press, 2006:596-9.

8. Rowe RC, Sheskey PJ, Owen SC (eds). Propylene glycol. In Handbook of pharmaceutical excipients. 5th edn. London: Pharmaceutical Press, 2006:521-3.

9. Eigenmann PA, Haenggeli CA. Food colourings, preservatives, and hyperactivity. Lancet 2007;370:1524-5.

10. Food Standards Agency. Update on food colours and hyperactivity, 5 November 2007. http://www.food.gov.uk/news/newsarchive/2007/nov/coloursmeeting (accessed 25 Nov 2008).

11. Joint FAO/WHO Expert Committee on Food Additives (JECFA). Evaluation of certain food additives and contaminants. World Health Organ Tech Rep Ser 1983;696:20.

12. Joint FAO/WHO Expert Committee on Food Additives (JECFA). Summary of evaluations performed by the Joint FAONWHO Expert Committee on Food Additives (JECFA) 1956-1995 (First Through Forty-fourth Meetings). Washington, DC: International Life Sciences Institute (ILSI) Press, 1996:14.

13. Department of Health. How much is too much? http//www.dh.gov.uk/en/ Publicationsandstatistics/Publications/PublicationsPolicyAndGuidance/DH 4139673 (accessed 25 Nov 2008).

14. Gray R, Henderson J. Review of the fetal effects of prenatal alcohol exposure. Report to the Department of Health. Oxford: National Perinatal Epidemiology Unit (NPEU), University of Oxford, 2006

15. BMA. Fetal alcohol spectrum disorders: a guide for health professionals. 2007. BMA Board of Science. www.bma.org.uk (accessed 25 Nov 2008). 
16. Department of Health. Updated alcohol advice for pregnant women. 2007. www. gnn.gov.uk (accessed Mar 2008).

17. Arulanantham K, Genel M. Central nervous system toxicity associated with ingestion of propylene glycol. J Pediatr 1978:93:515-16.

18. Martin G, Finberg L. Propylene glycol: a potentially toxic vehicle in liquid dosage form. J Pediatr 1970;77:877-8.

19. MacDonald MG, Getson PR, Glasgow AM, et al. Propylene glycol: increased incidence of seizures in low birth weight infants. Pediatrics 1987;79:622-5.

20. FAOMHO. Evaluation of certain food additives. Eighteenth Report of the Joint FAO/ WHO Expert Committee on Food Additives. World Health Organ Tech Rep Ser 1974;557:1-37

21. Committee on Nutrition American Academy of Pediatrics. The use and misuse of fruit juice in pediatrics. Pediatrics 2001;107:1210-13.
22. Duro D, Rising R, Cedillo M, et al. Association between infantile colic and carbohydrate malabsorption from fruit juices in infancy. Pediatrics 2002:109:797-805.

23. Beaugerie L, Lémann $M$, Jian $R$, et al. Effect of glucose and lipids on intestinal absorption of sorbitol: role of gastric emptying. Neurogastroenterol Motil 1996;8:235-9

24. Jauniaux E, Hempstock J, Teng C, et al. Polyol concentrations in the fluid compartments of the human conceptus during the first trimester of pregnancy: maintenance of redox potential in a low oxygen environment. J Clin Endocrinol Metab 2005;90:1171-5.

25. Brusati V, Józwik M, Józwik M, et al. Fetal and maternal non-glucose carbohydrates and polyols concentrations in normal human pregnancies at term. Pediatr Res 2005:58:700-4.

26. Brownlee M. Biochemistry and molecular cell biology of diabetic complications. Nature 2001:414:813-20.

\section{Images in neonatal medicine}

\section{Congenital subglottic cyst in a term neonate}

A full-term infant born via vaginal delivery developed inspiratory stridor shortly after birth. Routine resuscitation was performed at delivery without the need for intubation. He received supplemental oxygen for 1 day. His chest $x$ ray, airway fluoroscopy, contrast oesophagram and echocardiogram were unremarkable. Laryngoscopy and bronchoscopy revealed a large left anterolaterally based subglottic cyst obstructing $95 \%$ of the airway (figs 1A,B). The remainder of the airway appeared normal. The infant tolerated a transoral, endoscopic excision of the cyst. The cyst appeared to originate from a defect of the cricoid cartilage. Figure $1 \mathrm{C}$ reveals the post-surgical view of the airway. He successfully extubated on the morning following the surgery. A follow-up bronchoscopy 1 week and 3 months post-operatively revealed a normal airway with no evidence of recurrence (fig 1D).

Congenital subglottic cysts are very rare causes of neonatal stridor. ${ }^{1}$ Subglottic cysts are nearly always acquired and related to endotracheal intubation. Mucosal tissue damage is thought to lead to obstruction of mucous glands and cyst formation. Therefore, subglottic cysts tend to be seen in premature infants who require ventilator support. ${ }^{2}$ Our case is definitively congenital as there was no prior airway manipulation. A review of the literature revealed only two case reports of congenital subglottic cysts. ${ }^{3}{ }^{4}$ The presence of stridor in a neonate should lead one to think about a congenital airway abnormality and evaluation via direct laryngoscopy and rigid bronchoscopy should be considered.

\section{J Bruno, ${ }^{1}$ L P Smith, ${ }^{2}$ K B Zur, ${ }^{2}$ K C Wade ${ }^{1}$}

${ }^{1}$ Department of Pediatrics, Division of Neonatology, The Children's Hospital of Philadelphia, Philadelphia, USA; ${ }^{2}$ Department of Surgery, Division of Otolaryngology, The Children's Hospital of Philadelphia, Philadelphia, USA

Correspondence to: Kelly Wade, Division of Neonatology, The Children's Hospital of Philadelphia, 34th Street and Civic Center Blvd, Philadelphia, PA 19104, USA; kelly. wade@uphs.upenn.edu

Acknowledgements: We appreciate the willingness of the family to allow us to present this case.
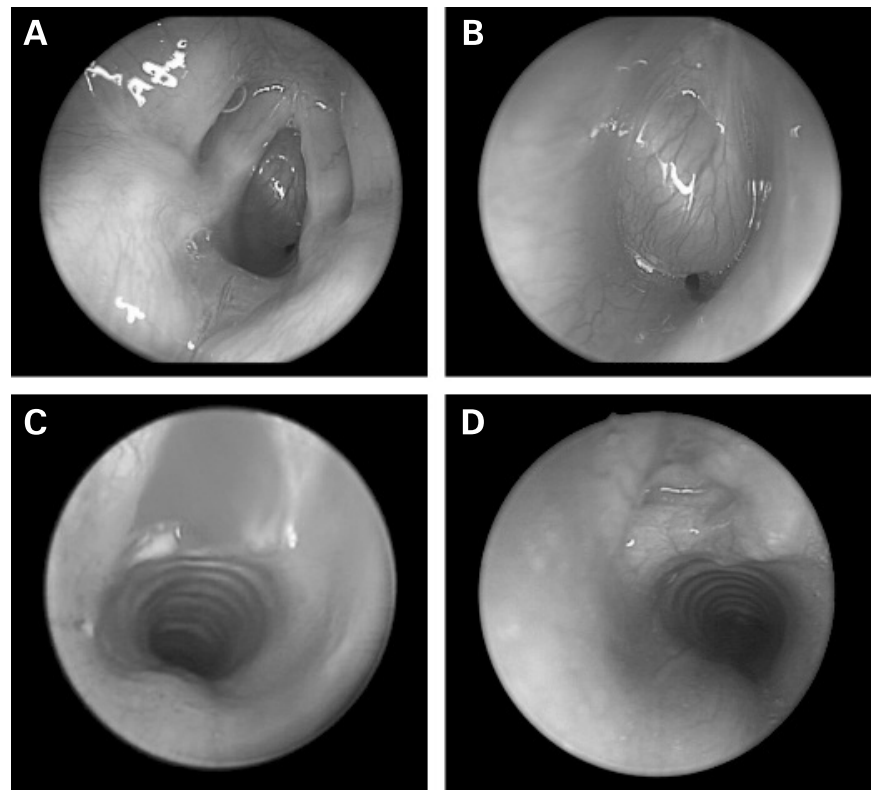

Figure 1 Congenital subglottic cyst. (A) View of large subglottic cyst just beneath the vocal cords. (B) Close-up view of the subglottic cyst. (C) Immediate post-excision view of the airway. (D) 3-month follow-up view of the airway with no evidence of recurrence.

\section{Competing interests: None.}

Ethics approval: This report was approved by the Children's Hospital of Philadelphia's Institutional Review Board.

Patient consent: Parental consent obtained.

Accepted 2 March 2009

Arch Dis Child Fetal Neonatal Ed 2009;94:F240. doi:10.1136/adc.2008.153502

\section{REFERENCES}

1. Pak MW, Woo JK, van Hasselt CA. Congenital laryngeal cysts: current approach to management. J Laryngol Otol 1996;110:854-6.

2. Couriel JM, Phelan PD. Subglottic cysts: a complication of neonatal endotracheal intubation? Pediatrics 1981;68:103-5.

3. Chamberlain D. Congenital subglottic cyst of the larynx. Laryngoscope 1970;80 254-9.

4. Sahhar HS, Marra S, Boyd C, et al. Ectopic subglottic thymic cyst: A rare cause of congenital stridor. Ear Nose Throat J 2003;82:873-4. 